\title{
Larval Development and Behavior in Early Life Stages of Black Sea Bream Reared in the Laboratory
}

\author{
Osamu Fukuhara* \\ (Accepted July 11, 1986)
}

\begin{abstract}
More than 2000 specimens reared in the laboratory were used to observe larval development and growth from hatching to about $10 \mathrm{~cm}$ long. Observations of moving time and swimming speed were made for larval and early juvenile stages. Morphological characters of fin development and pigmentation were illustrated in detail. Growth in length was expressed by the equation $Y=2.83$ $+0.15 X+0.00247 X^{2}$ for first 120 days, where $Y=$ standard length in $\mathrm{mm}$ and $X=$ days after hatching. Transformation from larvae to juveniles occurred during 9.0 to $11.0 \mathrm{~mm}$ standard length (SL). Swimming speed ranged from about $1.0 \mathrm{SL} / \mathrm{s}$ for larval stage to $2.0-2.5 \mathrm{SL} / \mathrm{s}$ for fish at metamorphosis. Various characters of digestive tract, squamation and pigment pattern as well as fins developed and differentiated when the larvae transformed to juveniles. The implication of organogenesis, morphogenesis and behavior in the early life stages was discussed.
\end{abstract}

Black sea bream Acanthopagrus schlegeli is an important sparid fish in fiheries and aquaculture in Japan. ${ }^{1)}$ Embryonic and larval development have been described briefly by Seno, ${ }^{2}$ Kishinouye, ${ }^{3)}$ Uchida et al. $^{4)}$ and Kasahara et al., ${ }^{\text {s) }}$ and Zhang et $a l{ }^{b)}$ Inspite of the importance of this species in fisheries and aquaculture, little information exists on behavior relative to initial feeding and swimming, growth and morphometric characters from larval through early juvenile stages. Tanaka $^{\text {? }}$ described the development of the alimentary canal, and development of digestive enzymes was reported by Kawai and Ikeda. ${ }^{8)}$ This paper provides comprehensive information on the early life history of laboratory reared $A$. schlegeli needed to better understand its biology and to produce the species in a large scale in hatchery for utilizing in aquaculture programs.

\section{Materials and Methods}

\section{Rearing Techniques}

Naturally spawned eggs were transferred from Hiroshima Municipal Fisheries Center to Nansei Regional Fisheries Research Laboratory on June 1, 1985. Transfer time was $45 \mathrm{~min}$. The eggs were immediately stocked in a rectangular tank $(1.0 \times 1.6 \times 0.7 \mathrm{~m}$ depth, $700 \mathrm{l}$ capacity $)$ at a density of about 5000 eggs/l. The temperature was initially $17^{\circ} \mathrm{C}$. Larvae were fed laboratoryreared Brachionus plicatilis for the first 25 days after hatching; thereafter Artemia nauplii was given.
Euphausia sp. and formulated pellets were used as weaning food. Cultured Chlorella $\mathrm{sp}$. was added daily in the rearing tank to provide food for rotifers (Fig. 1). A static water system was employed for the first 26 days; later a "flow through" system was used. Other techniques of larval rearing were similar to those described by Fukuhara. ${ }^{1)}$ The record of temperature, salinity and feeding schedule is shown in Fig. 1.

\section{Staryation Experiment}

The optimal time of initial feeding is required to make effective feeding by observing the behavior and mortality under unfed condition as well as fed condition. The time required to attain three developmental milestones, mouth opening, yolk absorption and oil globule absorption, was determined in separate experiments using a $30 /$ circular tank with a constant temperature of about $20^{\circ} \mathrm{C}$ in 1974. Sufficient larvae were maintained in the tank for daily measurement of growth and morphological observations. A $5 l$ glass container was also used to observe daily mortality of starved larvae at a constant temperature of $20^{\circ} \mathrm{C}$ in 1974.

\section{Larval Behavior}

New-born larvae were kept in a $30 l$ circular tank to observe larval behavior, particularly activity pattern. Swimming speeds of larvae were recorded every day in a $700 l$ tank, after they became active swimmers. The apparatus and methods at observation were identical to those of

* Nansei Regional Físheries Research Laboratory, Ohno, Saeki, Hiroshima 739-04, Japan (福原 修: 南西 海区水鷹研究所). 


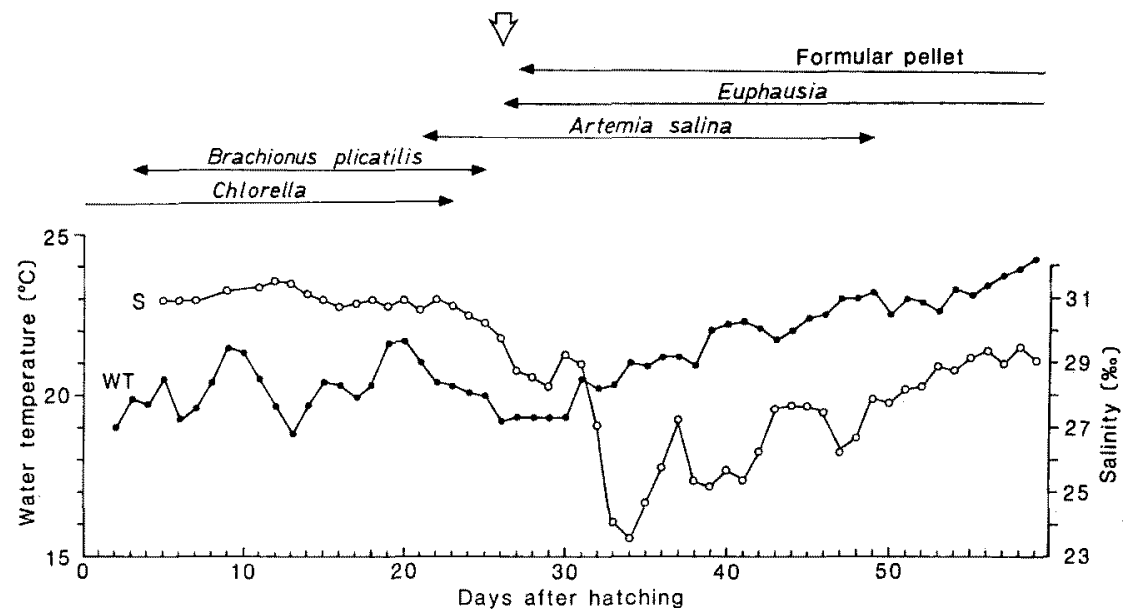

Fig. 1. Environmental conditions of water temperature, salinity and feeding periods of various prey used during the initial 60 days of the rearing experiment. Vertical arrow indicates the onset of a running water system.

Fukuhara.$^{9)}$

\section{Sampling of Specimens and Observation}

More than 35 larvae were collected from the rearing tank at random for the further observations on morphological characters and length measurement. The sampling was conducted every day for the initial 40 days, then at intervals of 4 or 5 days afterwards. Preservation and morphological observations of fish specimens were identical to those used for Pagrus major. ${ }^{9)}$

\section{Results}

\section{Eggs}

Fertilized eggs were spherical in shape, and contained a single oil globule. Perivitelline space was narrow. Naturally spawned eggs averaged $0.902 \mathrm{~mm}$ in diameter $(\mathrm{n}=114, \mathrm{SD}=0.027)$, with a range of 0.832 to $0.965 \mathrm{~mm}$. The eggs hatched 2 days after spawning at an incubation temperature of $17.2^{\circ} \mathrm{C}$.

\section{Development of Larvae and Juveniles}

New-born larvae $(\mathrm{n}=20)$ were $2.097 \mathrm{~mm} \pm \mathbf{0 . 0 5 4}$ SD in length (snout to tip of notochord) and $2.179 \mathrm{~mm} \pm 0.056 \mathrm{SD}$ in total length (snout to edge of caudal fin), and had a nearly spherical yolk sac with an oil globule at an anterior position (Fig. 2A). Growth in length of laboratory-reared larvae is given in Fig. 10. The mouth opened 3 days after hatching, with some yolk and oil globule still remaining. These were absorbed completely at 4 days and 5 days after hatching, respectively (Figs. 3, 7). Fed larvae began to feed on given prey of rotifer in a rearing tank prior to complete yolk absorption. Unfed larvae survived up to 9 days after hatching (Fig. 3). The optic vesicles became larger in size as the larvae grew (Fig. 2AF). Cartilaginous hypural elements appeared in larvae as small as $4.74 \mathrm{~mm}$ SL, aged 11 (Fig. 2F), and larvae formed the hypural elements increased with larval ages. Day on 17 all observed larvae finished the formation of the hypural elements (Fig. 4). Notochord flexion began on day 13 after hatching, as soon as the hypural bones appeared and the caudal fin was completely formed in about 10 days (Fig. 4). Myomeres were chevron-shaped until the hypural elements started to ossify (Fig. 2F), and became fully formed after notochord flexion was completed (Fig. 2I). The nostrils started to separate at about 23 days after hatching, and completed this process in all specimens observed by day 33 (Fig. 4). The preopercular spines developed in larvae of $6.1 \mathrm{~mm} \mathrm{SL}$ (Fig. 2G), and increased markedly in number after the metamorphosis. ${ }^{10)}$ Transformation to the juvenile stage occurred at a length of 9.0 to 11.0 $\mathrm{mm} \mathrm{SL}$. Scales first appeared on larvae as small as $10.2 \mathrm{~mm} \mathrm{SL}$, and larvae larger than $16.0 \mathrm{~mm}$ $\mathrm{SL}$ were fully scaled. The details of squamation were described by Fukuhara. ${ }^{1}$

\section{Fin Development}

The fin-fold was present in newly hatched larvae and remained uniform until fin rays started to 

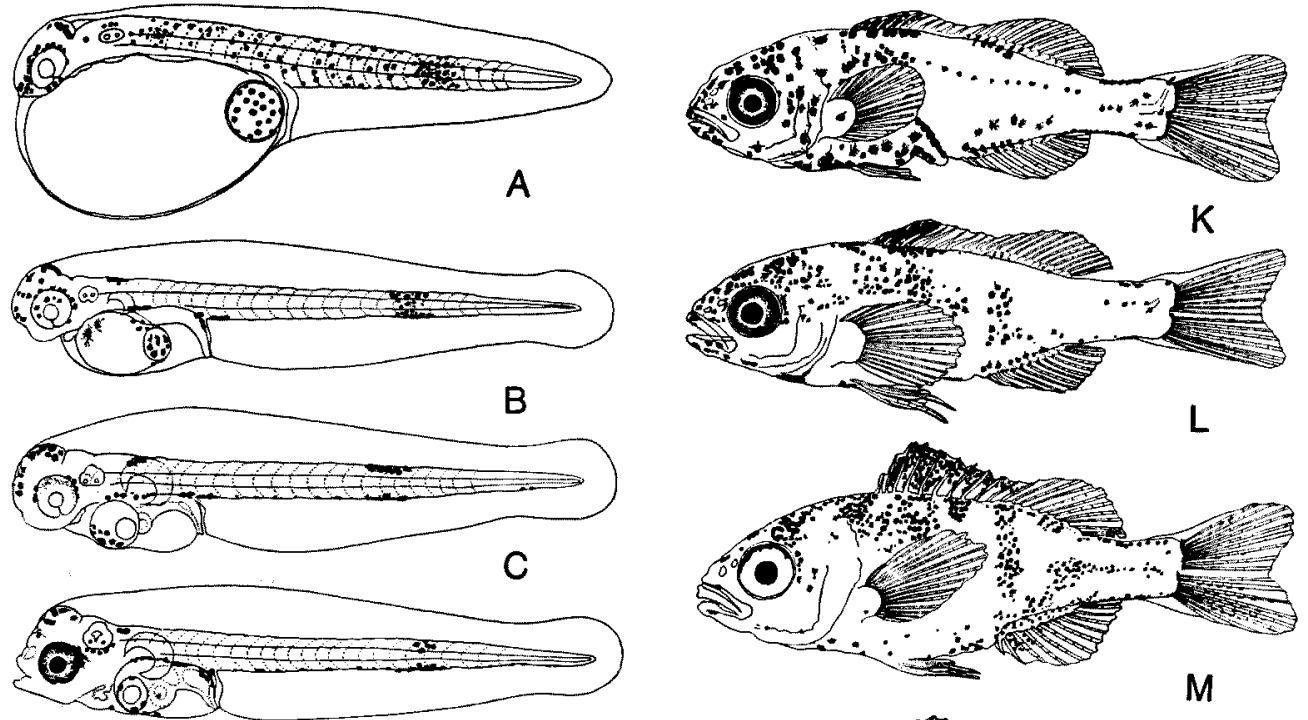

D

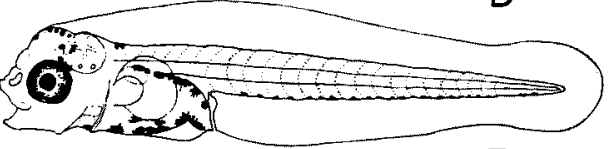

$E$
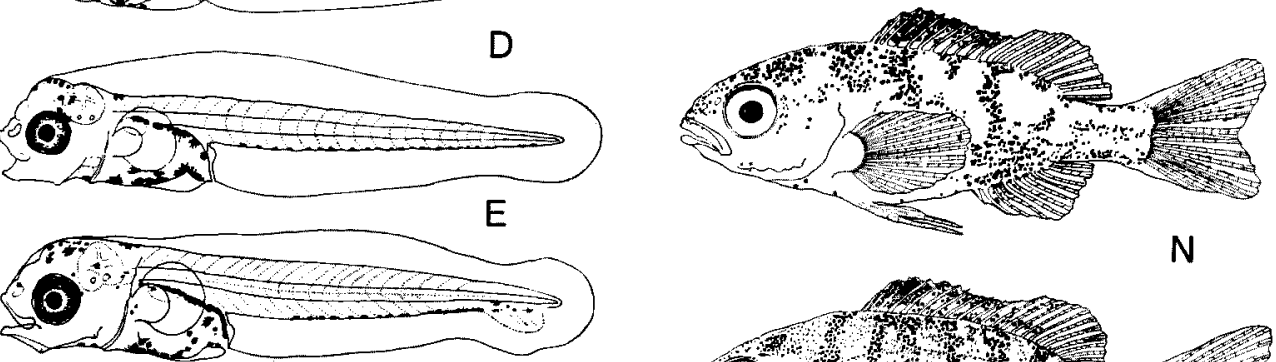

$\mathrm{F}$
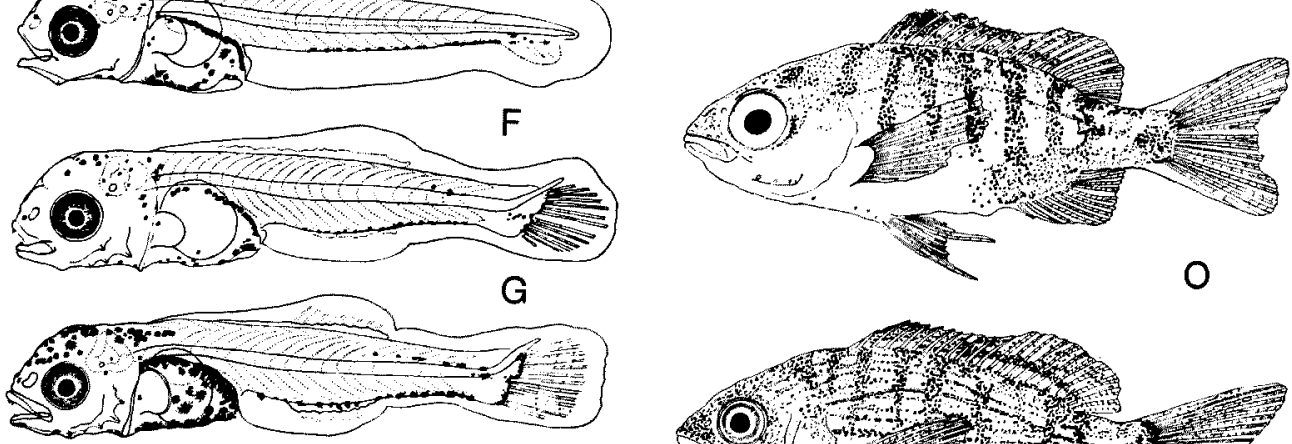

$\mathrm{H}$
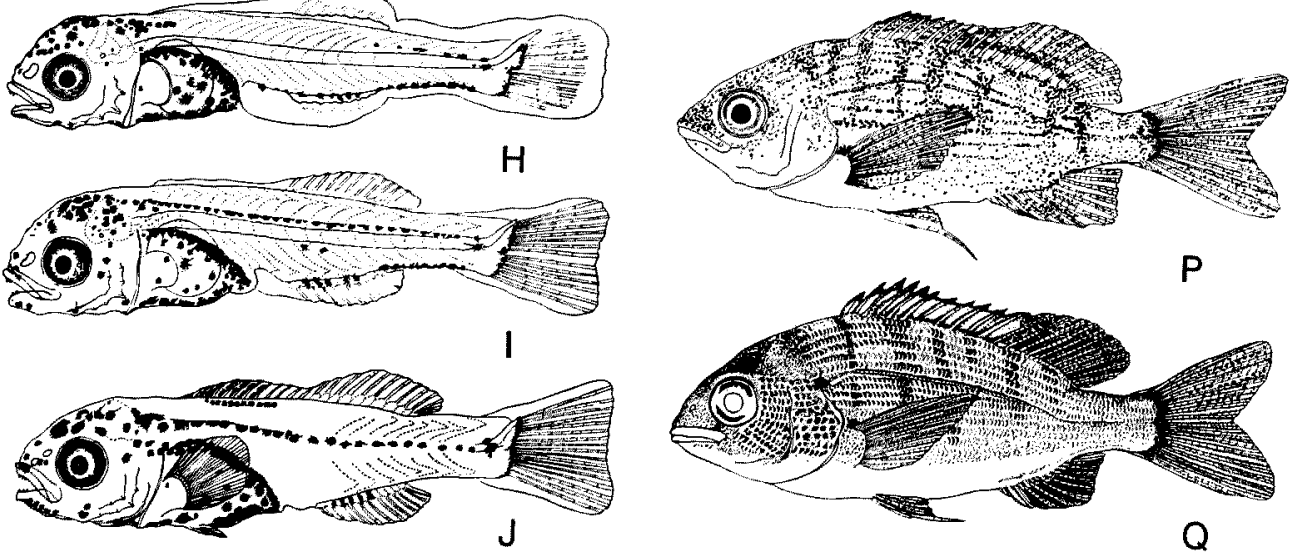

Fig. 2. Acanthopagrus schlegeli reared in the laboratory. A, $2.05 \mathrm{~mm} \mathrm{SL} ; \mathrm{B}, 2.80 \mathrm{~mm} \mathrm{SL} ; \mathrm{C}$, $2.95 \mathrm{~mm} \mathrm{SL}$; D, $3.09 \mathrm{~mm}$ SL; E, $3.46 \mathrm{~mm} \mathrm{SL} ;$ F, $4.74 \mathrm{~mm} \mathrm{SL}$; G, $6.1 \mathrm{~mm} \mathrm{SL}$; H, $6.7 \mathrm{~mm} \mathrm{SL;}$ I, 7.8 mm SL; J, $9.3 \mathrm{~mm} \mathrm{SL}$; K, $11.1 \mathrm{~mm} \mathrm{SL} ; \mathrm{L}, 12.0 \mathrm{~mm} \mathrm{SL}$; , $12.4 \mathrm{~mm} \mathrm{SL} ; \mathrm{N}, 13.9 \mathrm{~mm}$ SL; O, 17.8 mm SL; P, $28.7 \mathrm{~mm} \mathrm{SL;} \mathrm{Q,} \mathrm{73.6} \mathrm{mm} \mathrm{SL.}$ 
differentiate (Fig. 2G). Remnants of the larval fin-fold persisted until about $7.0 \mathrm{~mm}$ SL. The cartiliginous hypural elements were developed in larvae $4.74 \mathrm{~mm}$ long (Fig. 2F). Some segmented rays could be distinguished in larger larvae than $6.1 \mathrm{~mm}$ SL (Fig. 2G). The caudal fin was welldeveloped in larvae $7.8 \mathrm{~mm}$ SL (Fig. 2I).

A fan-shaped pectoral fin without rays was present in larvae of $2.80 \mathrm{~mm} \mathrm{SL}, 2$ days after hatching (Fig. 2B). The ventral fin buds appeared on 20-day old larvae, measuring $7.5 \mathrm{~mm}$ SL. Full fin ray complements for all fins were present in $9.3 \mathrm{~mm}$ SL larvae (Fig. 2J). Segmentation of fin rays was completed by $9.0 \mathrm{~mm}$ SL for the median fins and by $14.0 \mathrm{~mm}$ SL for the paired fins. Branching of fin rays started after segmentation was completed, and all fins were fully branched in larvae larger than $30 \mathrm{~mm} \mathrm{SL}$. ${ }^{1}$

The shape of the caudal fin was characterized by four phases during larval development; rounded, truncated, furcated and emarginated. The pectoral fin also changed in shape from fan-shaped to posteriorly pointed as development proceeded (Fig. 2).

\section{Pigmentation}

Newly hatched larvae had melanophores around the eyes, hind brain, on the oil globule and evenly in the trunk and caudal region. A band of melanophores was present on the thil at hatching (Fig. $2 \mathrm{~A}$ ), and remained until the larvae were about 3.0 mm SL, 4 days old (Fig. 2D). Serially arranged melanophores developed along the ventral midline of the tail. The melanophores became larger and more numerous in the gut area, especially in larvae

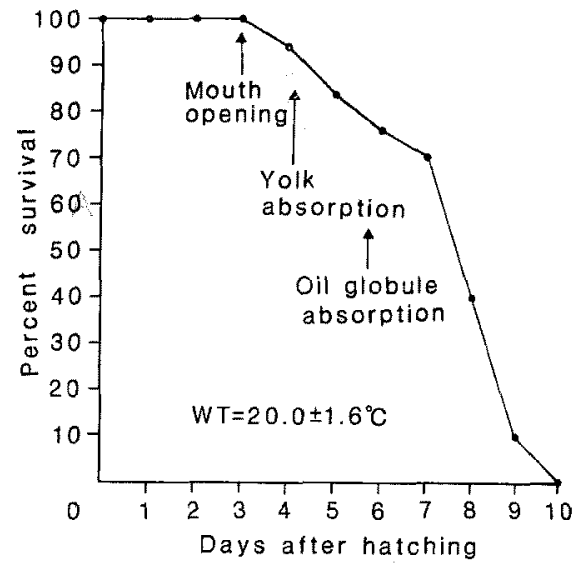

Fig. 3. Survival of unfed larvae of Acanthopagrus schlegeli. Initial number of individuals was 50.

between 6.7 and $9.3 \mathrm{~mm}$ SL (Fig. 2H-J). Relatively heavy pigment appeared in the head region and serially along the mid-lateral surface of the body after the larvae reached $7.8 \mathrm{~mm} \mathrm{SL}, 20$ days old (Fig. 2I, J). Newly transformed juveniles had evenly distributed melanophores on the caudal fin rays (Fig. 2J). Pigment bands developed in an anterior to posterior sequence beginning at the anterior portion of the trunk (Fig. $2 \mathrm{~K}-\mathrm{N}$ ); 7 or 8 bands were clearly distinguishable in specimens larger than $15 \mathrm{~mm} \mathrm{SL}$ (Fig. 2O). The sequence of pigmentation was described in detail by Fukuhara. ${ }^{1)}$ The pigment pattern of specimens larger than about $30 \mathrm{~mm}$ SL (Fig. 2P) became more obscure than the previous stages. The ray portions of all fins were heavily pigmented in speci-

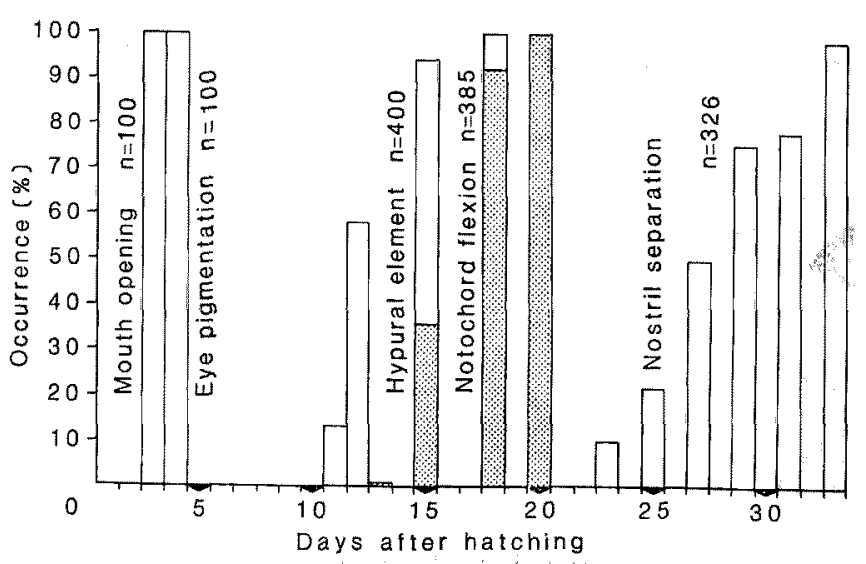

Fig. 4. Sequence of development in various morphological characters with larval ages in day after hatching for Acanthopagrus schlegeli. $\mathrm{n}=$ number of specimens observed. Dotted areas indicate developmental sequence of the notochord flexion. 


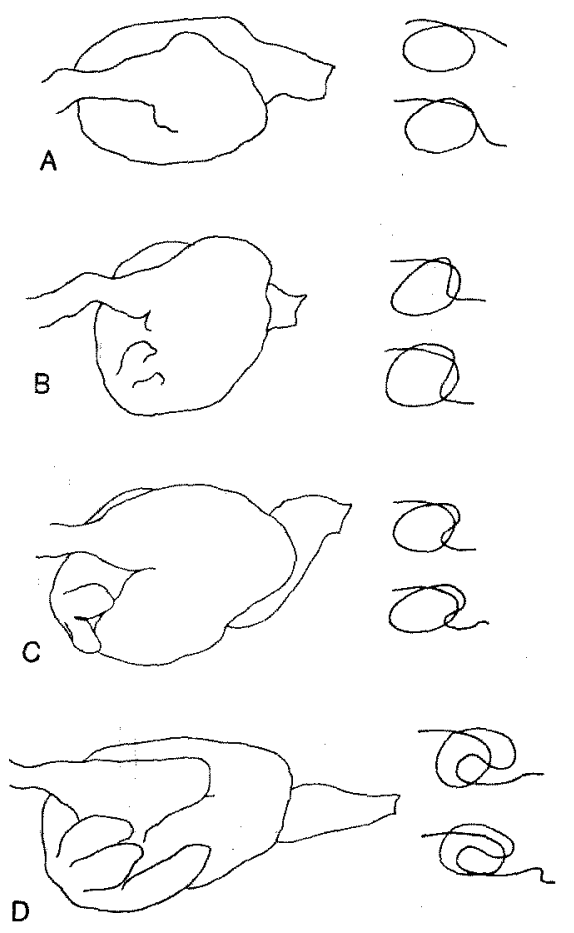

Fig. 5. Schematic illustration showing developmental stage of digestive organs in Acanthopagrus schlegeli.

mens over $28.7 \mathrm{~mm} \mathrm{SL}, 60$ days old (Fig. 2P, Q).

\section{The Development of Digestive Organ}

The sequence of development of the digestive organs during the larval to juvenile stage is given in Fig. 5. The digestive tract was coiled during larval stages as shown in Fig. 5A. Rudimentary pyloric caeca were present, and the posterior portion of the digestive tract assumed a rightangled configuration when the larvae transformed to early juvenile (Fig. 5B). The curvature then became more pronounced posteriorly than the previous stage, and the pyloric caeca developed well (Fig. 5C). As development proceeded, the pyloric caeca elongated, and the shape of the digestive tract became deeply rounded (Fig. 5D). Relationships between standard length, age and developmental stage of the digestive tract is shown Fig. 6. The development of the digestive tract was divided into 4 stages. Stage B, characterized larvae measuring $8.5 \mathrm{~mm} \mathrm{SL}, 25$ days old corresponded to the phase of transformation of larvae to juveniles. According to Tanaka, ${ }^{7,11}$ the differentiation of pyloric caeca is indicative of the transition from larvae to juvenile in various

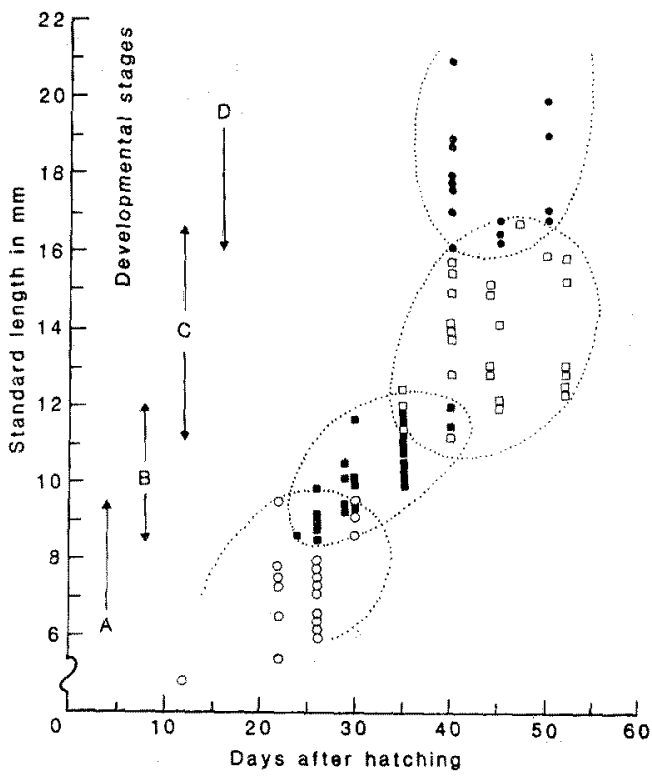

Fig. 6. Relations between developmental stages of digestive organs, standard length and age in Acanthopagrus schlegeli. The developmental stages $A, B, C$ and $D$ which are shown by open circles, closed rectangulars, open rectangulars and closed circles represent the developmental criteria depicted in Fig. 5, Dotted areas drawn freehand for showing the rough area of each developmental stage.

teleostean fishes including black sea bream, and also marks the completion of digestive tract development.

\section{Behavior}

Newly hatched larvae floated on the water surface of the tank. Usually the head was directed downward, and the larvae moved passively with the water currents caused by aeration. At the end of the yolk-sac period, larval locomotion increased sharply for both fed and unfed groups. Succeedingly active swimming was observed for fed larvae, and while the movement of unfed larvae declined after the yolk absorbed completely (Fig. 7). Vertical movement was dominant during early yolk sac stage, and this gradually transformed into horizontal movement during later stages, as was stated by Rosenthal and Hempel. ${ }^{12)}$ Active swimming consisting of feeding and searching behaviors differed from those observed in yolk-sac larvae on day 5 . In northern anchovy larvae ${ }^{18-14)}$ continuous swimming shifted to intermittent swimming when the yolk sac was 


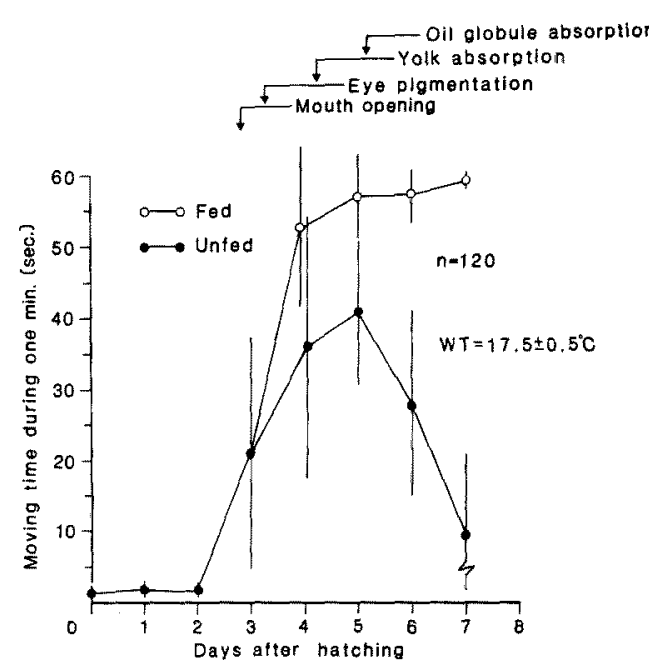

Fig. 7. Locomotion (length of swimming bursts per minute) during larval growth of Acanthopagrus schlegeli. Each point indicates the mean and standard deviation of 10 observations is shown by the bars. Note the difference of SD between fed and unfed groups.

absorbed at the third day of larval life. Swimming speed of larvae increased gradually as development proceeded during the first 2 weeks, then slightly decreased in the larvae after feeding began and then returned to the speed attained prior to initial feeding. A striking increment of swimming speed was detected for the larvae beyond day 26 , as they approached the transformation stage (Fig. 8). During larval stages, swimming speed was approximately 1 body length/s and $2-2.5$ body length/s up to 26 days.

\section{Growth and Allometric Growth}

Fed larvae grew steadily and no growth was observed in unfed larvae after yolk absorption (Fig. 9).

Larval growth for the initial 120 days is presented in Fig. 10. Overall growth could be expressed by the equation, $Y=2.83+0.15 X+2.47 \times 10^{-3} X^{2}$, where $Y=$ standard length, and $X=$ days after hatching. Larvae metamorphosed at about $\mathbf{9 . 0}$ $\mathrm{mm} \mathrm{SL}$, corresponding to day 28 after hatching.

The proportion of preanal length to total length was closely related to the developmental stage of the digestive organs. Relative preanal length increased from $30 \%$ to $50 \%$ while the digestive tract was developing during larval and early juvenile stages, and remained at $50 \% \mathrm{TL}$ after the

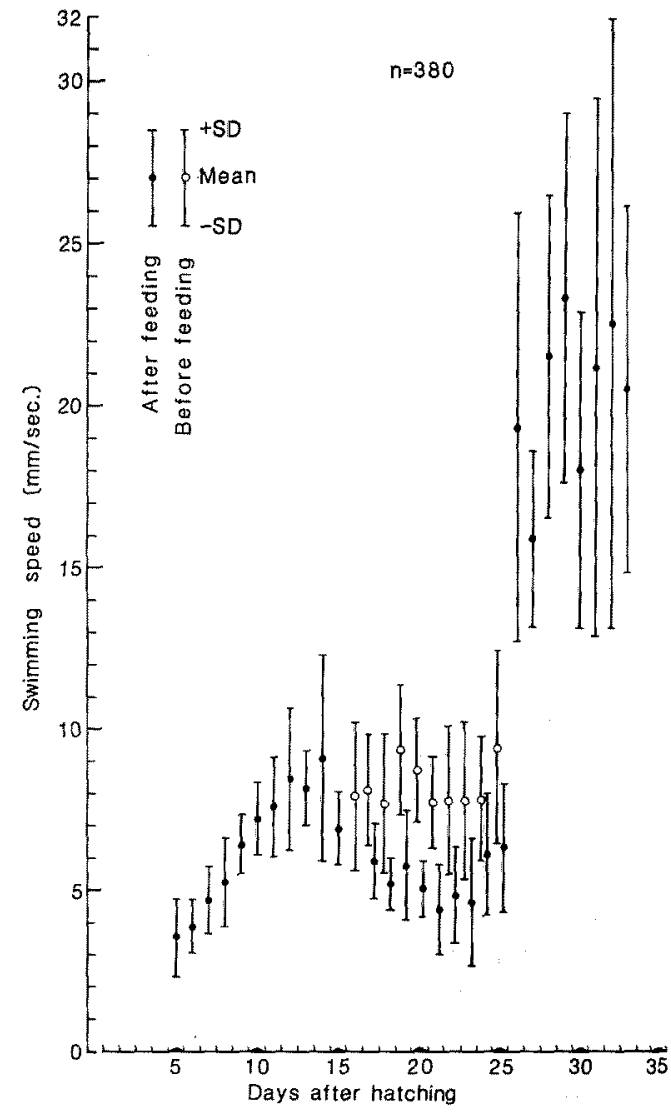

Fig. 8. Increment of swimming speed with larval growth in Acanthopagrus schlegeli, Each point represents 10 observations.

fish reached a length of $20 \mathrm{~mm} \mathrm{SL}$, and had an the adult-like stomach (Figs. 6, 11). Standard length was used to examine the development and growth of black sea bream with respect to other morphometric characters. The relationships between total length, standard length and preanal length could be expressed by linear regression (Fig. 12).

\section{Discussion}

During the course of larval life, the critical phase in physiological and behavioral development occurred between ages 3 and 4 day after hatching. With the shrinking of the yolk sac, the jaws became functional, the eyes became pigmented and locomotion increased, while the energy source changed from endogenous to exogenous. As for larval locomotion, swimming 


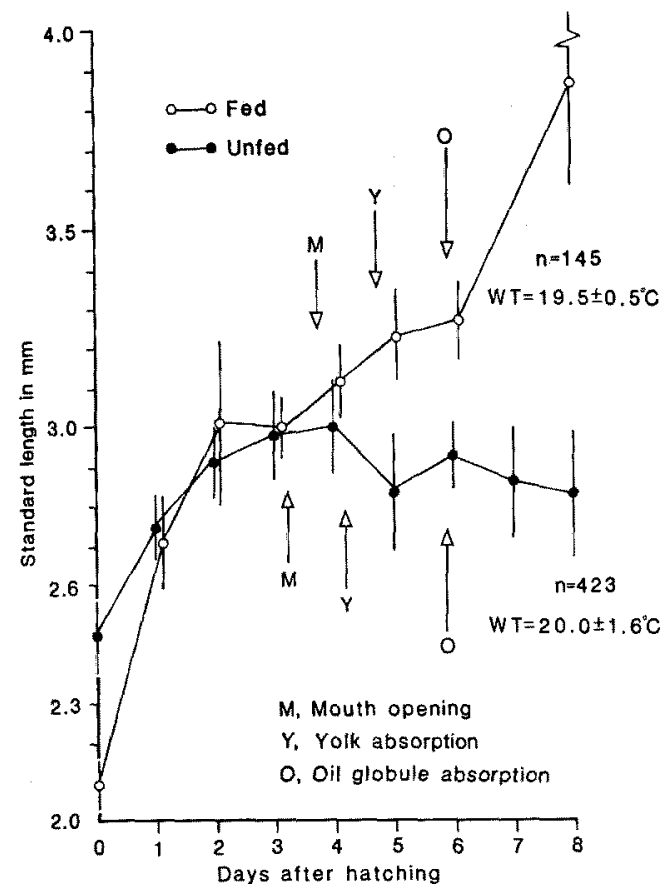

Fig. 9. Growth in length of fed and unfed larval Acanthopagrus schlegeli for the initial 8 days. Vertical arrow indicates the start of feeding. Points indicate mean and bars represent standard deviation.

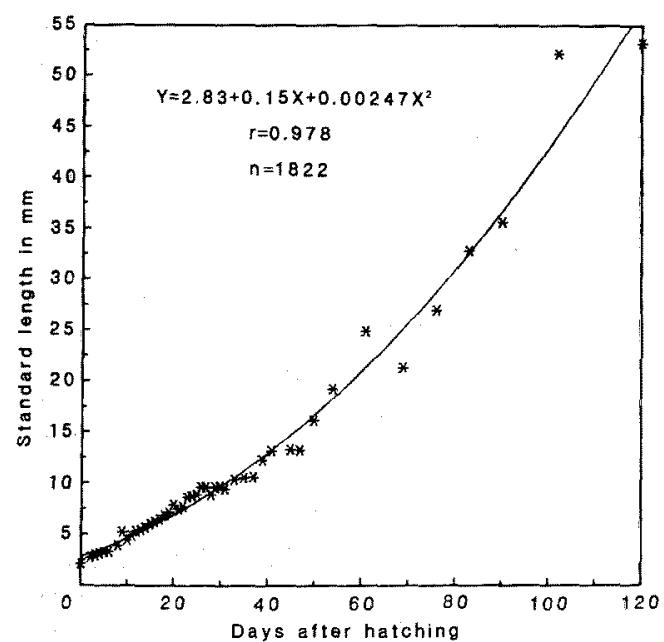

Fig. 10. Growth in length of larval Acanthopagrus schlegeli reared in the laboratory (temperature range $=18.8^{\circ}-25.9^{\circ} \mathrm{C}$ ). $\mathrm{r}=$ correlation coefficient; $\mathrm{n}=$ sample size.

changed drastically in mode and speed as mentioned before with changing the energy source.

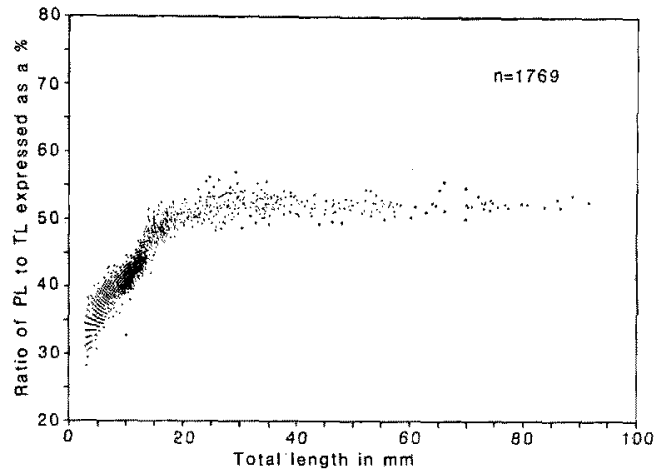

Fig. 11. Proportion of preanal length to total length for Acanthopagrus schlegeli larvae and juveniles.

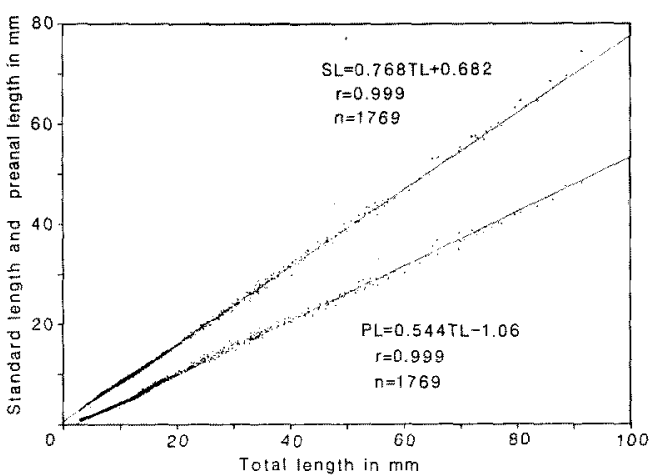

Fig. 12. Standard length (SL) and preanal length (PL), measuring from the tip of snout to the anus plotted against total length (TL) in artificially reared Acanthopagrus schlegeli.

This change of swimming mode has also been found in larvae of other marine fish species when the energy source changed from endogenous to exogenous. ${ }^{15,18)}$ Growth in length became maximum for unfed larvae and increased steadily for fed larvae while the yolk sac remained. Frequency of swimming bursts also reached a maximum with the new mode of swimming that accompanied yolk absorption. Reduction of growth and swimming activity were usually observed for unfed fish larvae after yolk absorption, ${ }^{17-18)}$ emphasizing the critical point associated with yolk absorption in larval life of black sea bream.

The sequence of development in various organs after yolk absorption is represented in Fig. 13. Transition from larvae to early juveniles occurred between 9.0 and $11.0 \mathrm{~mm} \mathrm{SL}$ in the reared specimens; in contrast this occurred in field specimens 


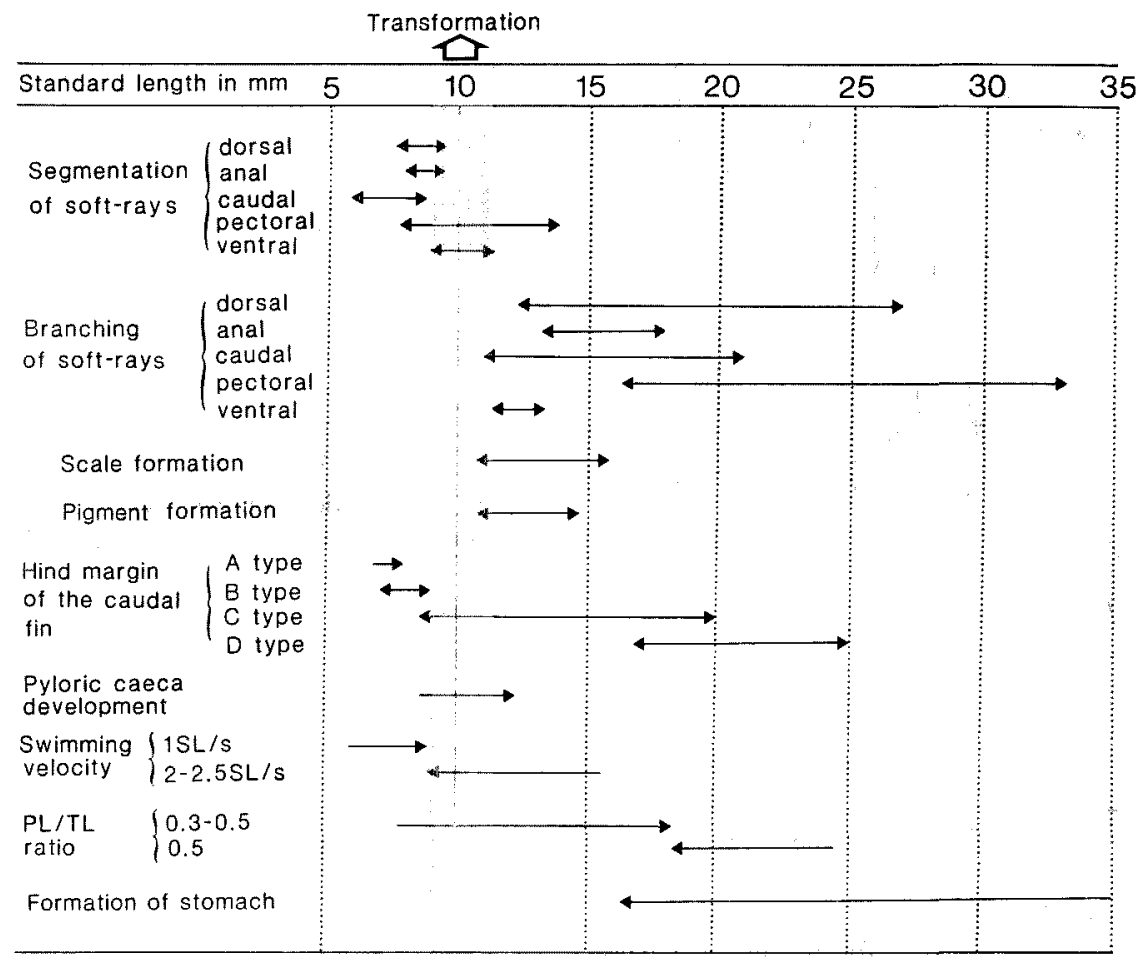

Fig. 13. The sequence of development of various morphological characters in the early life stages of Acanthopagrus schlegeli. Hind margin of the caudal fin; A, rounded; B, truncated; C, emarginated; D, furcated. PL, preanal length; TL, total length.

at about $8.0 \mathrm{~mm} \mathrm{SL.4)} \mathrm{No} \mathrm{available} \mathrm{information}$ exists for wild-caught specimens to compare with reared ones in subsequent developmental stages.

A rapid increase in swimming speed occurred coincidently with morphological development. Swimming speed of black sea bream in relation to larval size was similar to that of northern anchovy, ${ }^{13)}$ herring ${ }^{12)}$ and plaice, ${ }^{15)}$ but was slower than pacific mackerel. ${ }^{16)}$ This may be attributable to the completion of fin rays, especially in caudal fin. Formation of a truncated caudal fin was indicative for transformation of larval development in black sea bream as well as red sea bream. ${ }^{\text {p) }}$

The differentiation of the pyloric caeca and gastric gland was closely linked with the transition from larvae to juveniles. ${ }^{11,202}$ The development of the gastric gland was critical to the change of the digestive system required in changing from live plankters to a ground meal diet or formular pellet (refer to Fig. 1). In addition the initiation of squamation and band formation were observed after transformation. According to the field investigation, ${ }^{21)}$ juvenile black sea bream inhabit the Zostera zone after they attain $8.6 \mathrm{~mm}$ SL. Juveniles change prey preference from copepods to Caprella and/or Gammarus in the nature ${ }^{22}$ when fish attained a length of $20 \mathrm{~mm} \mathrm{SL}$, achieving constant ratio of PL/TL. These observations of development of both internal and external characters involving behavior suggested that the transformation occurring between 9.0 to $11.0 \mathrm{~mm}$ SL was accompanied by development of various organs as well as behaviors. Furthermore the coincidence of organogenesis resulted probably in the functional development, and leaded the shift of the life mode in early life stages of larval Acanthpagrus schlegeli. These findings suggest the possibility of the change in rearing procedures such as feeding items, water supply and transportation, and utilization of artificially reared seeds for releasing program after the fish grow up beyond the metamorphosis.

\section{Acknowledgements}

The author expresses sincere thanks to Professor T. Iwai, Kyoto University, for his suggestion and 
advice for the study. Thanks also due to Dr. H. Geoffrey Moser, Southwest Fisheries Center, National Marine Fisheries Service, NOAA, La Jolla, for his critical review of the manuscript. I am indebted to the Hiroshima Municipal Fisheries Center for help to collect the experimental materials.

\section{References}

1) O. Fukuhara: Bull. Nansei Reg. Fish. Res. Lab., No. 10, 1-16 (1977).

2) H. Seno: Zoological Magazine, 24, 195-197 (1912).

3) K. Kishinouye: Bull. Sci. Fish. Assac., 1, 185199 (1915).

4) K. Uchida, S. Imai, S. Mito, S. Fujita, M. Ueno, Y. Shojima, T. Senta, M. Tahuku, and Y. Dotu: Studies on the Eggs, Larvae and Juvenile of Japanese Fishes, Series 1. Second Lab. Fish. Biol., Fish. Dep. Fac. Agri. Kyushu Univ., 1958, pp. 66-73.

5) S. Kasahara, R. Hirano, and Y. Ohshima: Nippon Suisan Gakkaishi, 26, 239-244 (1960).

6) X. Zhang, G. He, and X. Sha: Acta Zoo. Sinica, 26, 331-336 (1980).

7) M. Tanaka: Studies on the structure and Function of the Digestive System of Teleostean Larvae. $\mathrm{Ph}$. D. Thesis, Kyoto University, Kyoto, 1973, pp. 1-136.
8) S. Kawai and S. Ikeda: Nippon Suisan Gakkaishi, 39, 877-881 (1973).

9) O. Fukuhara: Nippon Suisan Gakkaishi, 51, 731-743 (1985).

10) Y.U. Kim, R. Hirano, and S. Egusa: Publ. Mar. Lab. Pusan Fish. Coll. 4, 39-46 (1971).

11) M. Tanaka: Japan. J. Ichthyol., 18, 164-174 (1971).

12) H. Rosenthal and G. Hempel: in "Marine Food Chains" (ed. by J. H. Steele), Univ. Calif. Press, Berkeley, 1970, pp. 344-364.

13) J. R. Hunter: Fish. Bull., 70, 821-838 (1972).

14) P. W. Webb and R. T. Corolla: Fish. Bull., 79, 143-150 (1981).

15) J. H. S. Blaxter and M. E. Staines: in "Fourth European Marine Biology Symposium" (ed. by D. J. Crisp), Cambridge Univ. Press, London 1971 , pp. $467-485$.

16) J. R. Hunter and C. A. Kimbrell: Fish. Bull., 78, 89-101 (1980).

17) R. Lasker: Copeia, No. 2, 399-405 (1964).

18) K. Tsukamoto and T. Kajihara: Nippon Suisan Gakkaishi, 50, 59-61 (1984).

19) O. Fukuhara: Nippon Suisan Gakkaishi, 52, 8191 (1986).

20) J. J. Govoni: Rapp. P.-v. Reun. Cons. int. Explor. Mer, 178, 314-315 (1981).

21) Y. Ohsima: Nippon Suisan Gakkaishi, 10, 249255 (1942).

22) T. Fukuda: Rep. Inland Sea Fish. Res., 79-88 (1984). 